\title{
The Effect of Fatty Amine Chain Length on Synthesis Process of InP/ZnS Quantum Dots
}

\section{ZAHRA RANJBAR NAVAZI ${ }^{1}$, ALI NEMATI ${ }^{1 *}$, HAMID AKBARI ${ }^{2}$ and SOUDABEH DAVARAN ${ }^{3}$}

\author{
'Department of Material Engineering, Science and Research Branch, \\ Islamic Azad University, Tehran, Iran. \\ ${ }^{2}$ Faculty of Pharmacy, Tehran University of Medical Sciences (TUMS), Tehran , Iran. \\ ${ }^{3}$ School of Advanced Medical Sciences, Tabriz University of Medical Sciences, Tabriz, Iran. \\ ${ }^{*}$ Corresponding author E-mail: nemati.sharif.edu @ chmail.ir
}

http://dx.doi.org/10.13005/ojc/320446

(Received: July 26, 2016; Accepted: August 22, 2016)

\begin{abstract}
Obtaining narrow size distribution through conventional methods used for quantum dots of group II-VI semiconductors is impractical in the case of III-V semiconductors specially InP/ZnS quantum dots because of molecular precursors depletion and growth stage continuation through Ostwald ripening process. Using fatty amines as activator along with precursors can lead to more monodispersed quantum dots. In this work, the effect of fatty amine chain length on InP/ZnS quantum dots synthesis was investigated. Octylamine, dodecylamine and oleylamine were used as the activator of InP/ZnS quantum dots synthesis. Synthesis progress and color changes in reaction mixture with time lapse, indicative of formed quantum dots concentration, was intensified in presence of fatty amines with shorter chain length. Quantum dots with smaller mean size and broader size distribution were synthesized in presence of longer fatty amines as a result of higher capping capacity of them. Thereupon the optical properties of quantum dots were affected by chain length of fatty amine. Longer wavelength of photoluminescence emission was achieved by using octylamine with the shortest chain length among selected fatty amines.
\end{abstract}

Keywords: InP/ZnS Quantum Dots; fatty amines; octylamine; dodecylamine; oleylamine.

\section{INTRODUCTION}

Semiconductor nanocrystals, also called "Quantum dots", display unique optical properties due to quantum confinement effect as their size is smaller than Bohr exciton radius ${ }^{1}$. Exclusive properties of quantum dots have caused emersion of interest toward them in a variety of research fields specially in electronics, optoelectronics ${ }^{2}$, energy conversion ${ }^{3,4}$ and medicine ${ }^{5}$. Colloidal quantum dots have emerged as color tunable fluorescent probes in biological applications ${ }^{6,7}$. Large surface area of quantum dots has made them proper bio-functionalized nano-carrier for gene or drug delivery. Accordingly they can be used for simultaneous diagnosis and therapy ${ }^{8-13}$. However 
biological applications needs biocompatible and safe materials, so heavy metal-free quantum dots are preferred. InP/ZnS quantum dots are the most studied one among quantum dots with the least toxicity and prominent quantum confinement effect due to relatively large exciton radius $(15 \mathrm{~nm})$ as a result of robust covalent bonding and direct band gap of $\operatorname{InP}{ }^{14}$, but their synthesis is more difficult in compare with quantum dots of II-VI semiconductors to obtain monodisperse colloids of quantum dots ${ }^{15}$. Monodispersity of quantum dots is essential for attaining homogeneous physical and chemical properties. Broad size distribution of quantum dots is indication of heterogeneous properties specially photoluminescence emission of the quantum dots colloid ${ }^{16,17}$.

In order to control size distribution of quantum dots and obtain the desired size, mechanism of quantum dots formation should be comprehended. Quantum dots synthesis process include two primitive steps comprising nucleation and growth. Two main factors to be exerted during synthesis process for achieving narrow size distribution are separated nucleation and growth stages as well as diffusioncontrolled growth process. According to the GibbsTomson equation when the precursors monomer concentrations are lower than the threshold amount depending on the quantum dot size, Ostwald ripening occurs and leads to small particles dissolving and larger ones growing, whereby size distribution widening takes places. The growth mode is diffusioncontrolled when the size of the particles is larger than a critical value, so the smaller particles grow faster than larger ones and leads to size focusing ${ }^{18-22}$.

Capping ligands affect the size of quantum dots via alteration of monomers diffusion rate and activation energy ${ }^{23,24}$.

Fatty amines control the reaction rate of quantum dots formation ${ }^{25}$ and act as phosphorus precursor activator to generate reactive molecular kind $P$ that satisfy the requirements for obtaining size focusing effect in growth stage of quantum dots formation, as well as they can hinder the oxidation of Indium and improve the $\mathrm{ZnS}$ shell growth efficiency ${ }^{26}$, ${ }^{27}$. It has been confirmed that when primary fatty amines were used as ligand in $\mathrm{InP}$ quantum dots synthesis, heterogeneous growth of quantum dots from intermediate magic-sized clusters is switched to homogeneous growth from molecular precursors, resulting in narrower size distribution ${ }^{28}$.

In this research the effect of fatty amine chain length on InP/ZnS quantum dots size distribution and synthesis progress was investigated. It was shown that precursors reaction rate to form InP quantum dots, size distribution and mean size of colloidal quantum dots were impressed by structure of fatty amines.

\section{Experimental procedure}

\section{Materials}

Indium acetate, myristic acid, tristrimethylsilylphosphine $\left(\mathrm{P}(\mathrm{TMS})_{3}\right)$, oleylamine, sulfur and zinc acetate were purchased from Sigam Aldrich. Technical grade octadecene and octylamine were obtained from Merck. Dodecylamine was purchased from MP Biomedicals. All lab grade solvents including $n$-hexane, methanol, acetone, chloroform and ethanol were used as-received and without further purification. The synthesis process was adapted from previous reported literature ${ }^{27,31-33}$.

\section{(a)}

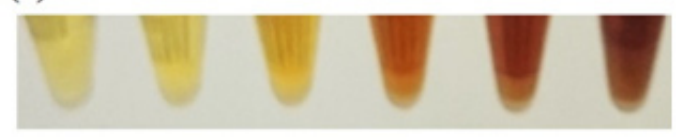

(b)

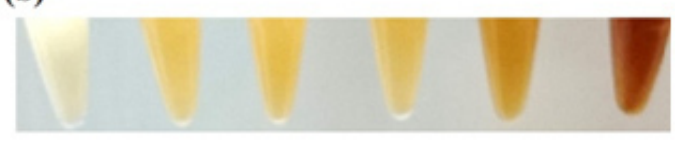

(c)

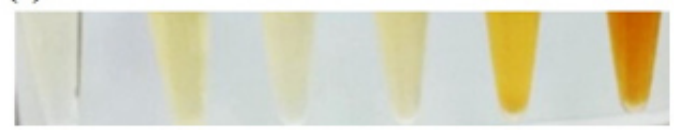

Fig. 1: InP quantum dots formation progress with time in reaction mixture containing (a) octylamine, (b)dodecylamine and (c)oleylamine after passing $10 \mathrm{~min}, 30 \mathrm{~min}, 60 \mathrm{~min}, 2 \mathrm{hr}$, $3 \mathrm{hr}$ and ZnS shell growth around InP core (left to right) 
InP/ZnS Colloidal Quantum Dots Synthesis Using Fatty Amines with Different Chain Lengths

For typical synthesis of InP/ZnS quantum dots, $0.4 \mathrm{mM}$ indium acetate, $1.4 \mathrm{mM}$ meristic acid and $5 \mathrm{cc}$ octadecene were mixed in a three- neck $50 \mathrm{cc}$ flask under stirring and heating up to 190 úc. The mixture was degassed for $1 \mathrm{hr}$ under argon flow. Separately precursor of phosphor was prepared using $0.2 \mathrm{mM}$ tristrimethylsilylphosphine $\left(\mathrm{P}(\mathrm{TMS})_{3}\right)$, $2.4 \mathrm{mM}$ fatty amine and $1 \mathrm{~g}$ octadecene as injection solution. After achieving clear solution and degassing the reaction mixture in 190 ÚC, the phosphor precursor was injected and caused temperature reduction in reaction mixture. The temperature was kept in 180 ÚC for the growth of nanocrystals nuclei. Aliquots of reaction mixture were taken at various time intervals for surveying growth of nanocrystals via UV-Vis absorption spectra and evident reaction mixture color change indicating the quantum dots formation reaction progress. Three samples with octylamine, dodecylamine and oleylamine were synthesized according to the mentioned procedure. After achieving the desired size of InP nanocrystals, precursors of zinc and sulfur were injected to the (a)

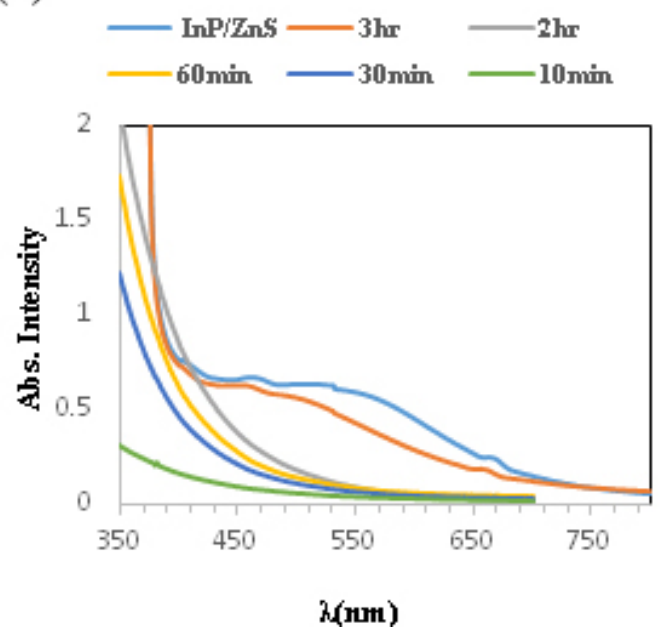

(b)

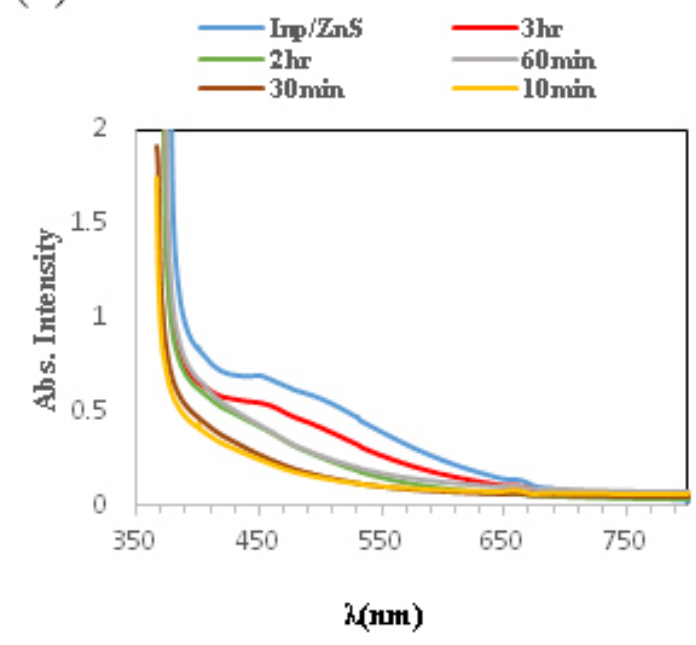

(c)

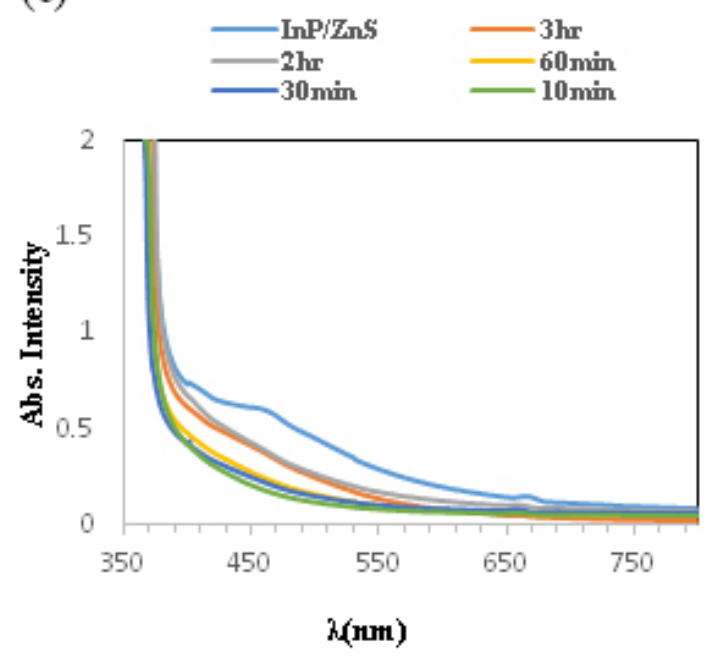

Fig. 2: Temporal shift of UV-Vis absorption spectra of InP/ZnS quantum dots synthesized using (a) octylamine, (b)dodecylamine and (c)oleylamine, as activator ligand 
reaction mixture for shell creation around nanocrystal core. For typical growth of $\mathrm{ZnS}$ shell, the reaction mixture temperature was reduced down to 150 ÚC and $1.5 \mathrm{cc}$ of $0.1 \mathrm{M}$ solution of zinc acetate in octadecene and $1.5 \mathrm{cc}$ of $0.1 \mathrm{M}$ sulfur solution in octadecene were injected in 150 ÚC with $10 \mathrm{~min}$ interval. Then the reaction mixture was heated up to 220 ÚC and kept 30 min in 220 ÚC for ZnS shell growth and again it was cooled down to 150 ÚC for injecting $1.5 \mathrm{cc}$ of zinc acetate and sulfur solutions as previous cycle. After finishing the shell growth step, the reaction mixture was cooled down to room temperature and quantum dots were purified using n-hexane and acetone followed by centrifugation for $20 \mathrm{~min}$ at $11000 \mathrm{rpm}$. The resulted red precipitate of the samples synthesized using different fatty amines can be redissolved in organic solvents such as toluene and chloroform for further characterization.

(a)

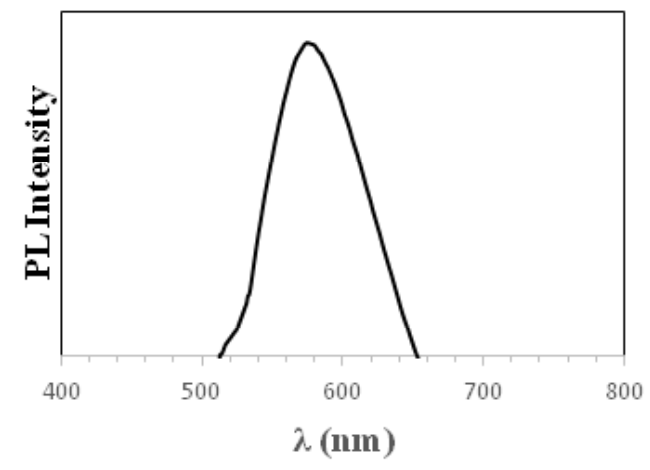

Absorption spectrum of quantum dots colloids sampling in different times and the final colloids containing core/ shell InP/ZnS quantum dots were obtained using Shimadzu 1650PC UV-vis spectrophotometer recorded from 300 to $800 \mathrm{~nm}$. Photoluminescence emission spectra of quantum dots synthesized in the presence of fatty amines with different chain lengths were investigated by Jasco FP-8000 spectrofluorometer at excitation wavelength of $400 \mathrm{~nm}$. The size and morphology of the sample with the most distinguishable absorption peak was determined by Zeiss EM 900 transmission electron microscope (TEM). The energy-dispersive $X$-ray spectrum of $\mathrm{InP} / \mathrm{ZnS}$ quantum dots for verification of chemical composition was obtained using Hitachi S4160 field emission scanning electron microscope (FESEM).

(b)

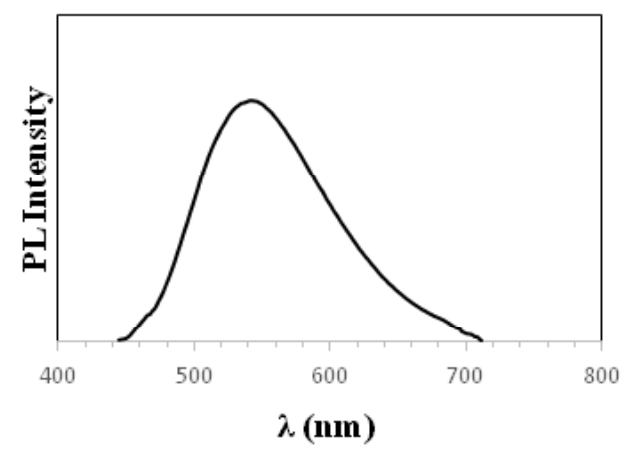

(c)

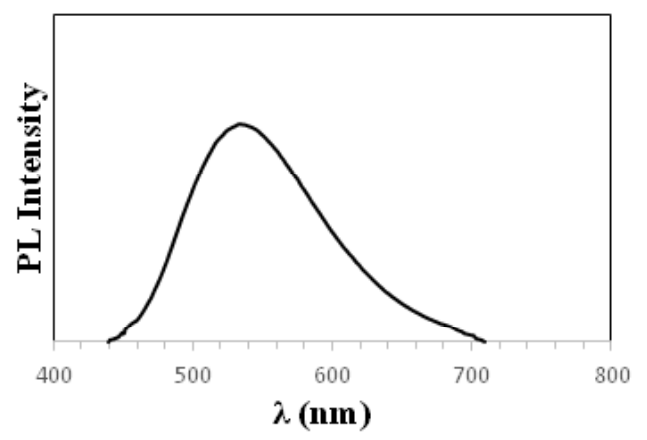

Fig. 3: Photoluminescence emission spectra of InP/ZnS quantum dots synthesized using (a) octylamine, (b) dodecylamine and (c) oleylamine 


\section{RESULTS AND DISCUSSION}

Reaction mixtures containing different fatty amines showed distinct color change with time indicating that fatty amine structure affect the formation progress of quantum dots. Figure 1 shows the color change trend in samples synthesized using octylamine (fig. 1a), dodecylamine (fig. 1b) and oleylamine (fig. 1c) representing the concentration of formed quantum dots as a result of reactive precursors interaction. According to figure 1, reaction progress in presence of oleylamine is slower in compare with others because of its longer chain length leading to higher temperature and prolix time lapse requirement. However using octylamine as ligand has led to faster reaction proceeding, thereupon yielding higher concentration of quantum dots as a result of shorter chain length and faster dissociation.

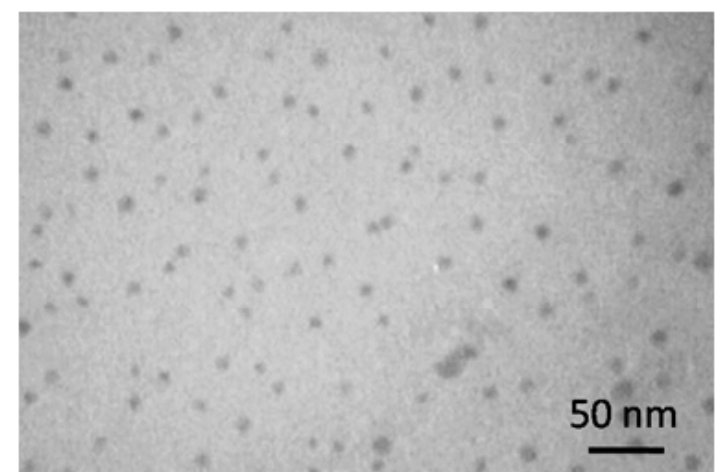

Fig. 5: EDS chemical analysis of InP/ZnS quantum dots synthesized using octylamine
Due to the low boiling point of fatty amines with shorter chain length than octylamine, they were not preferred as the temperature in $\mathrm{InP} / \mathrm{ZnS}$ quantum dots synthesis was 180 ÚC - 220 úC.

Figure 2 shows the evolution of UV-vis spectra of the samples with time, synthesized using octylamine, dodecylamine and oleylamine as ligand and activator in good accordance with apparent change in reaction mixture shown in figure 1.

According to absorption spectra of quantum dots in figure 2, using fatty amines with shorter chain length has led to more distinguishable absorption peak with longer wavelength, indicative of larger mean size of quantum dots. Higher activation energy and low reaction rate of amine with longer carbon chain leads to formation of smaller size quantum dots due to its higher capping capacity ${ }^{23}$. Hence octylamine yielded quantum dots with longer absorption wavelength as a result of lower capping capacity of it.

Photoluminescence emission spectra of InP/ZnS quantum dots synthesized using different fatty amines including octylamine, dodecylamine and oleylamine has been shown in figure 3 .

Full width at half maximum (FWHM) of emission spectrum indicated the size distribution of quantum dots, the smaller FWHM the narrower quantum dots size distribution ${ }^{29}, 30$. Actually wavelength of emitted light was impressed by the size of nanocrystal because of the band gap energy

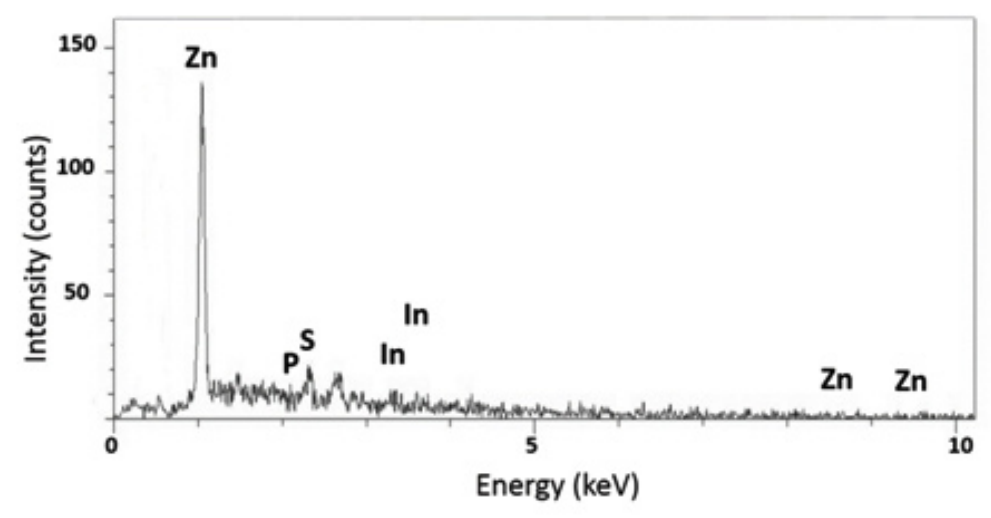

Fig. 4: TEM image of InP/ZnS quantum dots formed in presence of octylamine as activator ligand 
change as a result of quantum confinement effect. So the size distribution of quantum dots determined the wavelength of the emitted lights, thereupon the FWHM of the emission spectrum. As shown in figure 3 , there is difference between FWHM of emission spectra of quantum dots colloid synthesized in presence of fatty amines with different chain length. Actually the FWHM of photoluminescent emission spectra of quantum dots synthesized using octylamine, dodecylamine and oleylamine are respectively almost 75, 109 and $113 \mathrm{~nm}$. So using octylamine as precursors activator led to $\mathrm{InP} /$ ZnS quantum dots with narrower size distribution. A well as the maximum emitted wavelength of quantum dots was red shifted with applying shorter fatty amine. Accordingly quantum dots synthesized using octylamine as activator had the longest emitted wavelength as a result of larger average size of nanocrystals, more convenient for biological applications.

TEM image of InP/ZnS quantum dots synthesized using octylamine as activator in source solution of phosphor precursor has been shown in figure 4. According to figure 4 the average size of $\mathrm{InP} / \mathrm{ZnS}$ quantum dots was $10 \mathrm{~nm}$ with appropriate monodispersity and spherical morphology.

The results show that using fatty amines with shorter chain length are more convenient for achieving InP/ZnS quantum dots with larger mean size and narrower size distribution in defined time lapse.
Figure 5 shows EDS spectrum of synthesized quantum dots using octylamine as precursors activator, confirming chemical composition of $\mathrm{InP} / \mathrm{ZnS}$ quantum dots.

\section{CONCLUSION}

Synthesizing monodispersed colloidal quantum dots is essential to achieve homogenous optical properties. Fatty amines have been proved as precursors activator and diffusion rate controller, leading to quantum dots with narrower size distribution. The effect of fatty amine chain length on InP/ZnS quantum dots synthesis was explored. Size of quantum dots could be controlled not only by the precursors concentration but also by fatty amine structure and chain length. Slower color change of reaction mixture, indicative of concentration and size of formed quantum dots, occurred in the presence of fatty amine with longer chain length. We determined that shorter fatty amines were needed for obtaining higher concentration of formed quantum dots after defined time lapse with narrower size distribution and longer emission wavelength as a result of larger mean size of quantum dots, more favorable in biological applications.

\section{ACKNOWLEDGMENT}

This research was supported by Research Center for Pharmaceutical Nanotechnology, Tabriz University of Medical Sciences, Tabriz, Iran and I would like to thank all professors of this research center for their support and help. Also I would like to thank Dr. Mansour Akbari for his assistance with providing materials.

\section{REFERENCES}

1. Sargent, E.H., Nature photonics, 2012, 6(3), 133-135.

2. Bao, J. and M.G. Bawendi. Nature, 2015, 523(7558), 67-70.

3. Ning, Z., Nat Mater, 2014, 13(8), 822-828.

4. Chuang, C.-H.M., Nat Mater, 2014, 13(8), 796-801.

5. Voura, E.B., Nature medicine, 2004, 10(9), 993-998.

6. Medintz, I.L., Nature materials, 2005, 4(6), 435-446.
7. Zhang, C.-Y., Nature materials, 2005, 4(11),826-831.

8. Shi, D., Z. Guo, and N. Bedford, William Andrew Publishing: Oxford. 2015.

9. Rogach, A.L., Wien-New York: Springer, 2008.

10. Bruchez, M., Science, 1998, 281(5385), 2013-2016.

11. Choi, J. and N.S. Wang, INTECH Open Access Publisher. 2011.

12. Bolhassani, A. and S. Rafati, INTECH Open 
Access Publisher, 2011.

13. Zhang, Y. and A. Clapp., Sensors, 2011, 11(12), 11036-11055.

14. Wei, S., Journal of applied physics, 2004, 95(7), 3683-3688.

15. Liu, Z., Angewandte Chemie International Edition, 2008. 47(19), 3540-3542.

16. Sugimoto, T., Advances in Colloid and Interface Science, 1987, 28, 65-108.

17. Peng, Z.A. and X. Peng, Journal of the American Chemical Society, 2002, 124(13), 3343-3353.

18. Peng, X., J. Wickham, and A. Alivisatos, Journal of the American Chemical Society, 1998. 120(21), 5343-5344.

19. Kwon, S.G. and T. Hyeon, Small, 2011, 7(19), 2685-2702.

20. Dickerson, B.D., Virginia Polytechnic Institute and State University, 2005.

21. Shchukin, V., E. Sch, and P. Kratzer, , Springer, 2008

22. Battaglia, D. and X. Peng, Nano Letters, 2002, 2(9), 1027-1030.
23. Hwang, S., Japanese Journal of Applied Physics, 2010, 49(5S1), 05EA03.

24. Talapin, D.V., Colloids and Surfaces A: Physicochemical and Engineering Aspects, 2002, 202(2), 145-154.

25. Jeong, D.W., International Journal of Applied Ceramic Technology, 2015.

26. Song, W.-S., Journal of nanoparticle research, 2013, 15(6), 1-10.

27. Xie, R., D. Battaglia, and X. Peng, Journal of the American Chemical Society, 2007, 129(50), 15432-15433.

28. Gary, D.C., Chemistry of Materials, 2015, 27(4), 1432-1441.

29. Blackman, B.R., ProQuest, 2007.

30. Müllen, K. and U. Scherf, John Wiley \& Sons, 2006

31. Yong, K.-T., ACS nano, 2009, 3(3), 502-510.

32. Narayanaswamy, A., ACS nano, 2009, 3(9), 2539-2546.

33. Massadeh, S., S. Xu, and T. Nann. International Society for Optics and Photonics. 2009. 